Revta brasil. Bot., São Paulo, V.24, n.4, p.471-478, dez. 2001

\title{
Asteraceae do Parque Nacional da Serra da Canastra, Minas Gerais, Brasil ${ }^{1}$
}

\author{
JIMI NAOKI NAKAJIMA ${ }^{2,4}$ and JOÃO SEMIR ${ }^{3}$
}

(recebido: 22 de novembro de 2000; aceito: 20 de setembro de 2001)

\begin{abstract}
The Asteraceae in the Parque Nacional da Serra da Canastra, Minas Gerais, Brazil). A check-list of Asteraceae was carried out between February 1994 until January 1998, in Serra da Canastra National Park, which is located in Southern Brazil. All specimens are deposited at the herbarium of the Universidade Federal de Uberlândia, Minas Gerais, Brazil (HUFU). The Asteraceae is the most diverse family of the Serra da Canastra. The total number is 215 species, belonging to 66 genera, and 11 tribes. One significative result is the discovery of 27 new species, representing 19 genera of seven different tribes. Some of them belong to monotypic or small genera as Xerxes, Hololepis, Pseudobrickellia, and Inulopsis, and others to several genera which have great importance to the Brazilian Asteraceae flora, such as Lessingianthus, Chromolaena, Stevia, Mikania, Aspilia, Calea, and Senecio. This inventory has the highest number of species recorded in one study area in Brazil.
\end{abstract}

RESUMO - (Asteraceae do Parque Nacional da Serra da Canastra, Minas Gerais, Brasil). Uma listagem das espécies da família Asteraceae foi realizada no Parque Nacional da Serra da Canastra, situado no sudoeste do estado de Minas Gerais, entre fevereiro de 1994 a janeiro de 1998. Os exemplares coletados encontram-se depositados no herbário da Universidade Federal de Uberlândia, Minas Gerais (HUFU). Na Serra da Canastra, a família Asteraceae é a mais diversa em número de espécies com um total de 215 espécies, pertencentes a 66 gêneros e 11 tribos. As 27 espécies novas que foram descobertas pertencem a gêneros anteriormente monotípicos ou com poucas espécies, tais como Xerxes, Hololepis, Pseudobrickellia e Inulopsis, ou a vários gêneros com um grande número de espécies, tais como Lessingianthus, Chromolaena, Stevia, Mikania, Aspilia, Calea e Senecio. Este levantamento possui o maior número de espécies da família quando comparado com outras localidades no Brasil.

Key words: Asteraceae, Compositae, checklist, Serra da Canastra, Minas Gerais

\section{Introdução}

A família Asteraceae, com cerca de 1535 gêneros e aproximadamente 23000 espécies, representa cerca de $10 \%$ da flora mundial (Bremer 1994), e vem sendo intensivamente estudada nos últimos 25 anos não somente quanto à sua morfologia, anatomia, ontogenia e ecologia, mas também quanto a sua fitoquímica, citogenética e estrutura macromolecular (Bremer 1996, Hind \& Beentje 1996).

No Brasil, onde encontra-se boa parte da diversidade de Asteraceae, ainda são necessários levantamentos florísticos intensivos, uma vez que o monumental trabalho de Baker (1873-1884) é o último tratamento formal da família Asteraceae. Dentre os recentes levantamentos florísticos da família para um

1. Parte da tese de doutorado de J.N. Nakajima na Universidade Estadual de Campinas, SP, Brasil.

2. Universidade Federal de Uberlândia, Instituto de Biologia, Caixa Postal 593, 38400-902 Uberlândia, MG, Brasil.

3. Universidade Estadual de Campinas, Depto. de Botânica, Caixa Postal 6109, 13083-970 Campinas, SP, Brasil.

4. Autor para correspondência: nakajima@ufu.br determinado estado ou localidade, podem ser citados os de Harley \& Simmons (1986), Hind (1995), Moraes (1997), Dubs (1998) e Munhoz \& Proença (1998).

Em Minas Gerais, apesar da reconhecida importância da família Asteraceae na composição florística deste estado, existem apenas os levantamentos de Leitão Filho \& Semir (1987), Hind (1993) e Roque \& Pirani (1997). Na maior parte do estado não existe qualquer listagem ou tratamento das espécies, sendo este o caso do Parque Nacional da Serra da Canastra, o segundo maior parque da região Sudeste.

Neste parque existem apenas os poucos exemplares coletados no século passado entre 18191821 por Saint Hilaire (1847) em sua viagem para as nascentes do rio São Francisco, e as coletas ocasionais posteriores de outros botânicos mais recentemente. O desconhecimento sobre a composição florística deste importante parque nacional foi o principal motivo que levou a realização de um levantamento intensivo de sua flora fanerogâmica (Romero \& Nakajima 1999).

Desta forma, o presente trabalho tem por objetivo apresentar a listagem florística das espécies da família Asteraceae, e realizar análises florísticas com outras localidades já levantadas. 


\section{Material e métodos}

O Parque Nacional da Serra da Canastra, com uma área de 70525 hectares, situa-se na região Sudoeste do estado de Minas Gerais nos municípios de São Roque de Minas, Delfinópolis e Sacramento, nas coordenadas de $20^{\circ} 00^{\prime}$ $20^{\circ} 30^{\prime} \mathrm{S}$ e $46^{\circ} 15^{\prime}-47^{\circ} 00^{\prime} \mathrm{W}$ (Romero \& Nakajima 1999). As cotas altimétricas variam principalmente entre 800 $1000 \mathrm{~m}$. No divisor de águas das grandes bacias hidrográficas do rio São Francisco e do rio Paraná, a altitude média da Serra da Canastra atinge $1200 \mathrm{~m}$, com um máximo de $1496 \mathrm{~m}$ na Serra Brava (IBDF 1981).

O Parque está em uma zona de transição climática entre o clima tropical quente e o clima temperado mesotérmico. Acima de $1000 \mathrm{~m}$ ocorre um clima subtropical moderado úmido do tipo Cwb de Köppen, e abaixo de $1000 \mathrm{~m}$ ocorre um clima subtropical úmido do tipo Cwa de Köppen (IBDF 1981). Durante os quatro anos de coletas, as temperaturas médias máximas variaram de $18-31{ }^{\circ} \mathrm{C}$, e as mínimas de $9,5-17,5^{\circ} \mathrm{C}$. A pluviosidade variou de $0-1800 \mathrm{~mm}$, com chuvas iniciandose basicamente no mês de setembro e estendendo-se nos meses de outubro a fevereiro, com um decréscimo a partir do mês de março. O período de inverno é bastante seco, marcado por uma escassez de chuvas no mês de junho e um período de seca entre os meses de julho a meados de setembro (Romero 2000).

A área é caracterizada por dois grandes domínios geomorfológicos, os Remanescentes de Cadeias Dobradas, com dobras elaboradas em rochas proterozóicas, e a Região dos Planaltos da Canastra, com cristas, vales e barras na direção sudeste/noroeste. Em termos pedológicos, ocorre um predomínio de latossolos vermelho-amarelo distrófico, profundos e bem drenados, cambissolos distróficos, pouco desenvolvidos e moderadamente drenados, e solos litólicos distróficos, rasos e moderadamente drenados (IBDF 1981).

A vegetação é classificada em florestas semidecíduas, cerradão, cerrado, campo cerrado, campo e campo rupestre (IBDF 1981). Em um estudo de mapeamento da cobertura vegetal por meio de técnicas de sensoriamento remoto e trabalhos de campo, as seis fisionomias citadas acima foram detalhadas em 10 subtipos vegetacionais (Romero 2000), segundo o sistema de fitofisionomias proposto por Eiten (1983).

O levantamento florístico foi realizado de fevereiro de 1994 a janeiro de 1998, em intervalos de dois meses, totalizando 24 expedições, com duração de sete dias cada. Os locais de coleta foram estabelecidos de maneira a cobrir toda a extensão do Parque. Alguns locais, tais como a Serra do Cemitério, parte da Serra das Sete Voltas e o vale do Córrego do Coelho, não foram visitados devido à dificuldade de acesso.

Os exemplares coletados estão depositados no herbário da Universidade Federal de Uberlândia (HUFU) com duplicatas nos herbários F, R, UEC e US (siglas segundo Holmgren et al. 1990).

A identificação foi feita com base nas revisões e/ou estudos taxonômicos disponíveis da família, com o exame de exemplares-tipo ou de fotografias destes depositados nos herbários F, K e UEC, e com análise de exsicatas dos herbários ESA, F, NY, R, RB, SP, SPF, UEC e US (siglas segundo Holmgren et al. 1990).

A classificação adotada é a de Bremer (1994), que divide as Asteraceae em três subfamílias e 17 tribos. Em relação às tribos Eupatorieae e Vernonieae foram adotados os conceitos genéricos de King \& Robinson (1987) e Robinson (1999), respectivamente. Táxons infraespecíficos não foram considerados.

\section{Resultados e Discussão}

A família Asteraceae é a maior em número de espécies no Parque Nacional da Serra da Canastra (Nakajima 2000). Um total de 215 espécies foram levantadas, pertencentes a 66 gêneros de 11 tribos (tabela 1). As tribos mais ricas em espécies são Vernonieae (67), Eupatorieae (63) e Heliantheae (37). Por outro lado, a tribo Eupatorieae possui o maior número de gêneros (19), seguida por Vernonieae (16) e Heliantheae (10).

Os gêneros com mais espécies são Lessingianthus H. Rob. (27), Mikania Willd. (21), Baccharis L. (16), Chromolaena DC. (11) e Calea L. (10), cuja maioria das espécies é característica e representativa das diferentes fisionomias de cerrados e de campos rupestres de acordo com Goodland (1979), Eiten (1983) e Mendonça et al. (1998).

Um total de 27 espécies novas foram descobertas, pertencentes a 19 gêneros de sete tribos (tabela 1), o que corresponde a $13 \%$ do total das espécies de Asteraceae do parque. Algumas destas espécies novas são de gêneros monotípicos ou com poucas espécies, tais como Xerxes J.R. Grant, Hololepis DC. (Vernonieae), Pseudobrickellia R.M. King \& H. Rob. (Eupatorieae) e Inulopsis (DC.) O. Hoffman (Astereae), enquanto que outras são de gêneros bem representativos e de grande importância para a flora brasileira, tais como Lessingianthus (Vernonieae), Chromolaena, Stevia, Mikania (Eupatorieae), Calea, Aspilia (Heliantheae) e Senecio (Senecioneae).

Das 27 espécies novas, 19 são endêmicas na Serra da Canastra (tabela 1), além de outras três espécies anteriormente descritas (Calea brittoniana Pruski, Viguiera hilairei S.F. Blake e Stevia hilarii B.L. Rob.), totalizando uma taxa de $10 \%$ de endemismo. Algumas das espécies novas anteriormente reconhecidas como endêmicas por Romero \& Nakajima (1999) tiveram distribuições geográficas ampliadas para: (a) distribuição restrita ao sul e sudoeste de Minas Gerais e áreas limítrofes (Richterago campestris, Chresta sp. nov., Lessingianthus sp. nov. 5, Pseudobrickellia sp. nov. e Xerxes sp. nov.); (b) distribuição somente no estado de Minas Gerais (Lessingianthus sp. nov. 3); ou (c) distribuição no estado de Goiás (Richterago petiolata, Lessingianthus sp. nov. 1). Outras 30 espécies levantadas possuem um destes três tipos de distribuição geográfica (Nakajima 2000). 
Tabela 1. Lista das espécies de Asteraceae do Parque Nacional da Serra da Canastra, MG, de acordo com suas tribos (Bremer 1994). Os exemplares testemunhos estão sob os números de coleta de Rosana Romero (R) e de Jimi Naoki Nakajima (N). Os asteriscos representam as espécies endêmicas da Serra da Canastra, e as siglas as seguintes localidades da análise florística (SC

= Serra do Cipó; PA = Pico das Almas; AP = Alto Paraíso; GM = Grão-Mogol; $\mathrm{M}=$ Mucugê).

\begin{tabular}{|c|c|c|c|c|c|c|}
\hline & Coletor & $\mathrm{S} \mathrm{C}$ & PA & AP & GM & M \\
\hline \multicolumn{7}{|l|}{ BARNADESIEAE - 1 gênero, 3 espécies } \\
\hline Dasyphyllum flagellare (Casar.) Cabrera & R2316 & & & & & \\
\hline Dasyphyllum sprengelianum (Gardner) Cabrera & N1900 & $\mathrm{X}$ & $\mathrm{X}$ & & $\mathrm{X}$ & \\
\hline Dasyphyllum velutinum (Baker) Cabrera & N1337 & & & & & \\
\hline \multicolumn{7}{|l|}{ MUTISIEAE - 7 gêneros, 11 espécies } \\
\hline Actinoseris radiata (Vell.) Cabrera & R3270 & $\mathrm{X}$ & & & & \\
\hline Chaptalia integerrima (Vell.) Burkart & $\mathrm{N} 2830$ & $\mathrm{X}$ & & & & \\
\hline Gochnatia amplexifolia (Gardner) Cabrera & $\mathrm{R} 1117$ & $\mathrm{X}$ & & & & \\
\hline Gochnatia paniculata (Less.) Cabrera & R3863 & & & & & \\
\hline Holocheilus brasiliensis (L.) Cabrera & N2977 & & & & & \\
\hline Richterago campestris Roque \& J.N. Nakajima & N430 & & & & & \\
\hline Richterago petiolata Roque \& J.N. Nakajima & R3031 & & & & & \\
\hline Trixis glutinosa D. Don & $\mathrm{R} 2303$ & $\mathrm{X}$ & & $\mathrm{X}$ & & \\
\hline Trixis nobilis (Vell.) Katinas & R919 & & & & & \\
\hline Trixis vauthieri DC. & R4331 & $\mathrm{X}$ & $\mathrm{X}$ & & $\mathrm{X}$ & $\mathrm{X}$ \\
\hline Wunderlichia mirabilis Riedel ex Baker & R2499 & & & $\mathrm{X}$ & $\mathrm{X}$ & \\
\hline \multicolumn{7}{|l|}{ LACTUCEAE - 1 gênero, 1 espécie } \\
\hline Hypochaeris gardneri Baker & $\mathrm{R} 1288$ & $\mathrm{X}$ & & & & \\
\hline \multicolumn{7}{|l|}{ VERNONIEAE - 16 gêneros, 67 espécies } \\
\hline Acilepdopsis echitifolia (Mart. ex DC.) H. Rob. & R3538 & & & & & \\
\hline Centratherum punctatum Cass. & R3428 & $\mathrm{X}$ & & & & \\
\hline Chresta plantaginifolia (Less.) Gardner & N713 & $\mathrm{X}$ & & & & \\
\hline Chresta scapigera (DC.) Gardner & N1937 & & & $\mathrm{X}$ & & \\
\hline Chresta souzae H. Rob. & R893 & & & $\mathrm{X}$ & & \\
\hline Chresta sphaerocephala DC. & $\mathrm{R} 1058$ & & & & & \\
\hline Chresta sp. nov. & R948 & & & & & \\
\hline Chrysolaena herbacea (Vell.) H. Rob. & $\mathrm{R} 1322$ & & & & & \\
\hline Chrysolaena sp. nov. * & $\mathrm{R} 4577$ & & & & & \\
\hline Echinocoryne schwenkiifolia (Mart. ex DC.) H. Rob. & N1806 & $\mathrm{X}$ & & & & \\
\hline Echinocoryne stricta (Gardner) H. Rob. & R2296 & & & & & \\
\hline Elephantopus biflorus (Less.) Sch. Bip. & $\mathrm{R} 2302$ & & & & & \\
\hline Elephantopus micropappus Less. & $\mathrm{R} 1974$ & & & $\mathrm{X}$ & & \\
\hline Elephantopus riparius Gardner & $\mathrm{R} 2563$ & & & & & \\
\hline Eremanthus elaeagnus (Mart. ex DC.) Sch. Bip. & N1915 & & & & $\mathrm{X}$ & \\
\hline Eremanthus erythropappus (Sch. Bip.) MacLeish \& N. Schumach. & $\mathrm{R} 1136$ & $\mathrm{X}$ & & & & \\
\hline Eremanthus glomerulatus Less. & $\mathrm{R} 2204$ & & & $\mathrm{X}$ & & \\
\hline Eremanthus seidelii MacLeish \& N. Schumach. & N978 & & & & & \\
\hline Hololepis pedunculata (DC. ex Pers.) DC. & R658 & $\mathrm{X}$ & & & & \\
\hline Hololepis sp. nov. $1 *$ & N1289 & & & & & \\
\hline Hololepis sp. nov. $2 *$ & N2671 & & & & & \\
\hline Lepidaploa barbata (Less.) H. Rob. & $\mathrm{R} 2837$ & $\mathrm{X}$ & & & & \\
\hline Lepidaploa psilostachya (DC.) H. Rob. & R853 & $\mathrm{X}$ & & & & \\
\hline Lepidaploa rufogrisea (A. St. Hil.) H. Rob. & $\mathrm{R} 2012$ & $\mathrm{X}$ & & $\mathrm{X}$ & $\mathrm{X}$ & \\
\hline Lepidaploa sororia (DC.) H. Rob. & $\mathrm{R} 2385$ & & & & & \\
\hline Lessingianthus argyrophyllus (Less.) H. Rob. & N860 & & & $\mathrm{X}$ & & \\
\hline Lessingianthus bardanioides (Less.) H. Rob. & N1678 & & & $\mathrm{X}$ & & \\
\hline Lessingianthus buddleifolius (Mart. ex DC.) H. Rob. & R3329 & $\mathrm{X}$ & & $\mathrm{X}$ & & \\
\hline Lessingianthus cephalotes (DC.) H. Rob. & $\mathrm{N} 2858$ & & & & & \\
\hline Lessingianthus compactiflorus (Mart. ex Baker) H. Rob. & $\mathrm{R} 1850$ & & & & & \\
\hline Lessingianthus desertorum (Mart. ex DC.) H. Rob. & R3765 & $\mathrm{X}$ & $\mathrm{X}$ & & & \\
\hline Lessingianthus exiguus (Cabrera) H. Rob. & N1671 & & & & & \\
\hline Lessingianthus glabratus (Less.) H. Rob. & R3317 & & & & & \\
\hline Lessingianthus grandiflorus (Less.) H. Rob. & $\mathrm{R} 4578$ & & & & & \\
\hline Lessingianthus irwinii (G.M. Barroso) H. Rob. & $\mathrm{N} 2148$ & & & & & \\
\hline
\end{tabular}


(cont.)

\begin{tabular}{|c|c|c|c|c|c|c|}
\hline & Coletor & $\mathrm{SC}$ & PA & AP & GM & M \\
\hline Lessingianthus lacunosus (Mart. ex DC.) H. Rob. & N1680 & & & & & \\
\hline Lessingianthus ligulifolius (DC.) H. Rob. & $\mathrm{R} 1849$ & & & & & \\
\hline Lessingianthus linearifolius (Less.) H. Rob. & $\mathrm{R} 842$ & $\mathrm{X}$ & & $\mathrm{X}$ & & \\
\hline Lessingianthus linearis (Spreng.) H. Rob. & $\mathrm{R} 1030$ & $\mathrm{X}$ & & $\mathrm{X}$ & & $\mathrm{X}$ \\
\hline Lessingianthus pulverulentus (Baker) H. Rob. & R694 & & & & & \\
\hline Lessingianthus pumillus (Vell.) H. Rob. & R2041 & & & & & \\
\hline Lessingianthus roseus (Mart. ex DC.) H. Rob. & N1681 & $\mathrm{X}$ & & & & \\
\hline Lessingianthus simplex (Less.) H. Rob. & N5 10 & $\mathrm{X}$ & $\mathrm{X}$ & & & \\
\hline Lessingianthus tomentellus (Mart. ex DC.) H. Rob. & N2306 & $\mathrm{X}$ & & & & \\
\hline Lessingianthus virgulatus (Mart. ex DC.) H. Rob. & R2746 & $\mathrm{X}$ & & & & \\
\hline Lessingianthus warmingianus (Baker) H. Rob. & N1586 & $\mathrm{X}$ & & & & \\
\hline Lessingianthus sp. nov. 1 & R2842 & & & & & \\
\hline Lessingianthus sp. nov. $2 *$ & $\mathrm{R} 1028$ & & & & & \\
\hline Lessingianthus sp. nov. 3 & N887 & & & & & \\
\hline Lessingianthus sp. nov. $4 *$ & R3125 & & & & & \\
\hline Lessingianthus sp. nov. 5 & R3647 & & & & & \\
\hline Lessingianthus sp. nov. $6 *$ & R1796 & & & & & \\
\hline Lychnophora ericoides Mart. & $\mathrm{R} 1650$ & & & $\mathrm{X}$ & $\mathrm{X}$ & \\
\hline Lychnophora syncephala (Sch. Bip.) Sch. Bip. & $\mathrm{R} 1922$ & & & & & \\
\hline Lychnophora sp. nov. * & R3575 & & & & & \\
\hline Piptocarpha axillaris (Less.) Baker & $\mathrm{R} 2273$ & & & & & \\
\hline Piptocarpha macropoda DC. & R3625 & & & & & \\
\hline Piptocarpha oblonga Baker & N2602 & & & & $\mathrm{X}$ & \\
\hline Stenocephalum megapotamicum (Spreng.) Sch. Bip. & N2383 & $\mathrm{X}$ & & & & \\
\hline Stenocephalum tragiifolia (DC.) Sch. Bip. & N496 & & & & & \\
\hline Strophopappus speciosus (Less.) R. Esteves & N1216 & & & & & \\
\hline Vernonanthura membranacea (Gardner) H. Rob. & N1749 & & & & & \\
\hline Vernonanthura phosphorica (Vell.) H. Rob. & N1254 & & $\mathrm{X}$ & & & \\
\hline Vernonanthura rigiophylla (Kuntze) H. Rob. & N1214 & & & & & \\
\hline Vernonanthura viscidula (Less.) H. Rob. & $\mathrm{R} 1060$ & & & & & \\
\hline Vernonanthura westiniana (Less.) H. Rob. & R3847 & & & & & \\
\hline Xerxes sp. nov. & N1822 & & & & & \\
\hline \multicolumn{7}{|l|}{ PLUCHEAE - 1 gênero, 1 espécie } \\
\hline Pterocaulon rugosum Malme & R4069 & & & & & \\
\hline \multicolumn{7}{|l|}{ GNAPHALIEAE - 3 gêneros, 4 espécies } \\
\hline Achyrocline alata DC. & N1812 & $\mathrm{X}$ & $\mathrm{X}$ & $\mathrm{X}$ & $\mathrm{X}$ & \\
\hline Achyrocline satureioides DC. & R3483 & $\mathrm{X}$ & $\mathrm{X}$ & & $\mathrm{X}$ & \\
\hline Chionolaena sp. nov. * & R4139 & & & & & \\
\hline Lucilia lycopodioides (Less.) S.E. Freire & $\mathrm{R} 1056$ & & $\mathrm{X}$ & & & \\
\hline \multicolumn{7}{|l|}{ ASTEREAE - 4 gêneros, 20 espécies } \\
\hline Baccharis aphylla DC. & R3601 & & $\mathrm{X}$ & & & \\
\hline Baccharis brachylaenoides DC. & N2641 & & & $\mathrm{X}$ & & \\
\hline Baccharis calvescens DC. & $\mathrm{R} 4275$ & & $\mathrm{X}$ & & & \\
\hline Baccharis dracunculifolia DC. & R4066 & $\mathrm{X}$ & & & & \\
\hline Baccharis erigeroides DC. & R4866 & & & & & \\
\hline Baccharis gracilis DC. & R3775 & $\mathrm{X}$ & & & & \\
\hline Baccharis helichrysoides DC. & R3316 & & & & & \\
\hline Baccharis humilis Sch. Bip. ex Baker & R2784 & & & & & \\
\hline Baccharis ligustrina DC. & N2056 & $\mathrm{X}$ & $\mathrm{X}$ & $\mathrm{X}$ & & \\
\hline Baccharis pentziifolia Sch. Bip. ex Baker & N1522 & & & & & \\
\hline Baccharis linearifolia (Lam.) Pers. & $\mathrm{N} 2202$ & & & & & \\
\hline Baccharis schultzii Baker & R4313 & & & & & \\
\hline Baccharis subdentata DC. & $\mathrm{R} 1057$ & $\mathrm{X}$ & & $\mathrm{X}$ & $\mathrm{X}$ & \\
\hline Baccharis tarchonanthoides DC. & N2739 & & & & & \\
\hline Baccharis trimera DC. "sensu lato" & N388 & $\mathrm{X}$ & & & & \\
\hline Baccharis varians Gardner & N292 & & & $\mathrm{X}$ & & \\
\hline Conyza primulifolia (Lam.) Cuatrec. \& Lourteig & R4184 & & & & & \\
\hline Inulopsis camporum (Gardner) G.L. Nesom & N154 & $\mathrm{X}$ & & & & \\
\hline Inulopsis sp. nov. * & N691 & & & & & \\
\hline
\end{tabular}




\begin{tabular}{|c|c|c|c|c|c|c|}
\hline & Coletor & $\mathrm{SC}$ & PA & AP & GM & M \\
\hline Podocoma bellidioides Baker & R1553 & & & & & \\
\hline \multicolumn{7}{|l|}{ SENECIONEAE - 2 gêneros, 6 espécies } \\
\hline Hoehnephytum trixoides (Gardner) Cabrera & N1364 & $\mathrm{X}$ & & & $\mathrm{X}$ & $\mathrm{X}$ \\
\hline Senecio adamantinus Bong. & $\mathrm{R} 879$ & $\mathrm{X}$ & & $\mathrm{X}$ & $\mathrm{X}$ & \\
\hline Senecio brasiliensis Spreng. ex Baker & R4641 & & & & & \\
\hline Senecio emiliopsis C. Jeffrey & R4845 & & & & & \\
\hline Senecio pohlii Sch. Bip. ex Baker & R603 & $\mathrm{X}$ & & $\mathrm{X}$ & $\mathrm{X}$ & \\
\hline Senecio sp. nov. $*$ & R1069 & & & & & \\
\hline \multicolumn{7}{|l|}{ TAGETEAE - 2 gêneros, 2 espécies } \\
\hline Porophyllum angustissimum Gardner & N1041 & & & $\mathrm{X}$ & & \\
\hline Tagetes minuta $\mathrm{L}$ & R2111 & & & & & \\
\hline \multicolumn{7}{|l|}{ HELIANTHEAE - 10 gêneros, 37 espécies } \\
\hline Acanthospermum australe (Loefl.) Kuntze & N672 & & $\mathrm{X}$ & $\mathrm{X}$ & & \\
\hline Aspilia foliacea (Spreng.) Baker & N2925 & & & $\mathrm{X}$ & & $\mathrm{X}$ \\
\hline Aspilia laevissima Baker & N522 & $\mathrm{X}$ & & & & \\
\hline Aspilia reflexa Baker & R3136 & & & & & \\
\hline Aspilia riedelii Baker & R977 & & & & & \\
\hline Aspilia sp. nov. * & R1639 & & & & & \\
\hline Bidens flagellaris Baker & N1692 & & & & & \\
\hline Bidens graveolens Mart. & R1918 & & & $\mathrm{X}$ & & \\
\hline Bidens segetum Mart. ex Colla & N992 & & & & & \\
\hline Calea brittoniana Pruski * & $\mathrm{R} 850$ & & & & & \\
\hline Calea clausseniana Baker & $\mathrm{R} 885$ & & & & & \\
\hline Calea cuneifolia DC. & R4539 & & & & & \\
\hline Calea fruticosa (Gardner) Urbatsch, Zlotsky \& Pruski & N2255 & & & & & \\
\hline Calea graminifolia Sch.Bip. ex Krasch. & R646 & & & & & \\
\hline Calea multiplinervia Less. & R4748 & & & & & \\
\hline Calea myrtifolia Benth. \& Hook. f. & N2974 & & & & & \\
\hline Calea ramosissima Baker & R661 & & & & & \\
\hline Calea uniflora Less. & $\mathrm{R} 1366$ & & & & & \\
\hline Calea sp. nov. * & R4942 & & & & & \\
\hline Clibadium armanii (Balb.) Sch. Bip. ex O.E. Schulz & R3318 & & & $\mathrm{X}$ & & \\
\hline Dimerostemma asperatum S.F. Blake & R1949 & & & & & \\
\hline Dimerostemma humboldtianum (Gardner) H. Rob. & R3658 & & & & & \\
\hline Dimerostemma vestitum (Baker) S.F. Blake & $\mathrm{R} 4320$ & & & & & \\
\hline Ichthyothere integrifolia (DC.) Baker & N2861 & & & & & \\
\hline Ichthyothere mollis Baker & R4661 & & & & & \\
\hline Ichthyothere sp. nov. * & R4594 & & & & & \\
\hline Isostigma megapotamicum (Spreng.) Sherff & R4680 & $\mathrm{X}$ & & & & \\
\hline Viguiera discolor Baker & $\mathrm{R} 1550$ & & & & & \\
\hline Viguiera grandiflora Gardner & R900 & & & & & \\
\hline Viguiera hilairei S.F. Blake * & N886 & & & & & \\
\hline Viguiera macrocalyx S.F. Blake & R4207 & & & & & \\
\hline Viguiera robusta Gardner & R934 & & & & & \\
\hline Viguiera tenuifolia Gardner & N460 & & & & & \\
\hline Wedelia macedoi $\mathrm{H}$. Rob. & $\mathrm{R} 1172$ & & & & & \\
\hline Wedelia puberula DC. & R4852 & & & & & \\
\hline Wedelia subvelutina DC. & N1982 & & & & & \\
\hline Wedelia sp. nov. * & R3409 & & & & & \\
\hline \multicolumn{7}{|l|}{ EUPATORIEAE - 19 gêneros, 63 espécies } \\
\hline Ageratum fastigiatum (Gardner) R.M. King. \& H. Rob. & $\mathrm{R} 872$ & $\mathrm{X}$ & $\mathrm{X}$ & $\mathrm{X}$ & & \\
\hline Austrocritonia velutina (Gardner) R.M. King. \& H. Rob. & R4463 & & & & & \\
\hline Ayapana amygdalina (Lam.) R.M. King. \& H. Rob. & N1359 & $\mathrm{X}$ & $\mathrm{X}$ & & $\mathrm{X}$ & \\
\hline Barrosoa betoniciformis (DC.) R.M. King. \& H. Rob. & R3972 & & & & & \\
\hline Campuloclinium hirsutum Gardner & N1687 & & & & & \\
\hline Campuloclinium megacephalum (Mart. ex Baker) R.M. & & & & & & \\
\hline King. \& H. Rob. & R3854 & & & $\mathrm{X}$ & & \\
\hline Campuloclinium sp. nov. $*$ & N1732 & & & & & \\
\hline Chromolaena barbacensis (Hieron.) R.M. King. \& H. Rob. & R 1928 & $\mathrm{X}$ & & & $X$ & \\
\hline
\end{tabular}




\begin{tabular}{|c|c|c|c|c|c|c|}
\hline & Coletor & $\mathrm{SC}$ & PA & AP & GM & M \\
\hline Chromolaena campestris (DC.) R.M. King. \& H. Rob. & R891 & & & & & \\
\hline Chromolaena chasea (B.L. Rob.) R.M. King. \& H. Rob. & R3343 & & & & & \\
\hline \multicolumn{7}{|l|}{ Chromolaena cinereo-viridis (Sch. Bip. ex Baker) R.M. } \\
\hline King. \& H. Rob. & R3354 & & & & & \\
\hline Chromolaena costatipes (B.L. Rob.) R.M. King. \& H. Rob. & N514 & & & & & \\
\hline \multicolumn{7}{|l|}{ Chromolaena cylindrocephala (Sch. Bip. ex Baker) R.M } \\
\hline King. \& H. Rob. & N2331 & & & & & \\
\hline Chromolaena decumbens Gardner & R1667 & $\mathrm{X}$ & & & & \\
\hline Chromolaena stachyophylla (Spreng.) R. M. King. \& H. Rob. & $\mathrm{R} 1522$ & $\mathrm{X}$ & $\mathrm{X}$ & & & \\
\hline Chromolaena vindex (DC.) R.M. King. \& H. Rob. & R3767 & & & & & \\
\hline Chromolaena xylorhiza (Sch. Bip. ex Baker) R.M. King. \& H. Rob. & R3323 & & & & & \\
\hline Chromolaena sp. nov. * & R3870 & & & & & \\
\hline Disynaphia halimifolia (DC.) R.M. King. \& H. Rob. & R688 & & & & & \\
\hline Eupatorium lineatum Sch. Bip. ex Baker & $\mathrm{R} 1537$ & & & & & \\
\hline Grazielia dimorpholepis (Baker) R.M. King. \& H. Rob. & R1659 & & & & & \\
\hline Grazielia gaudichaudiana (DC.) R.M. King. \& H. Rob. & R4058 & & & & & \\
\hline Grazielia intermedia (DC.) R.M. King. \& H. Rob. & R2058 & & & & & \\
\hline Heterocondylus alatus (Vell.) R.M. King. \& H. Rob. & R3608 & $\mathrm{X}$ & & & & \\
\hline Heterocondylus decipiens (Baker) R.M. King. \& H. Rob. & R3256 & & & $\mathrm{X}$ & & \\
\hline Heterocondylus pandurifolius (Baker) R.M. King. \& H. Rob. & $\mathrm{R} 887$ & & & & & \\
\hline Koanophyllum adamantium (Gardner) R.M. King. \& H. Rob. & R1108 & & $\mathrm{X}$ & & $\mathrm{X}$ & \\
\hline Mikania banisteriae DC. & N1714 & & & & & \\
\hline Mikania decumbens Malme & $\mathrm{R} 865$ & & & & & \\
\hline Mikania dentata Spreng. & N2430 & & & & & \\
\hline Mikania laevigata Sch. Bip. ex Baker & N1969 & & & & & \\
\hline Mikania lasiandrae DC. & N1737 & & & $\mathrm{X}$ & & \\
\hline Mikania lindbergii Baker & N2352 & & & & & \\
\hline Mikania microcephala DC. & N2617 & & & & & \\
\hline Mikania mosenii Malme & R3328 & & & & & \\
\hline Mikania neurocaula DC. & N360 & & & & & \\
\hline Mikania nummularia DC. & R1026 & & & & & \\
\hline Mikania oblongifolia DC. & N1934 & $\mathrm{X}$ & & & & \\
\hline Mikania officinalis Mart. & N516 & $\mathrm{X}$ & $\mathrm{X}$ & & & \\
\hline Mikania purpurascens Sch. Bip. ex Baker & R680 & & & & & \\
\hline Mikania ramosissima Gardner & R4266 & & & $\mathrm{X}$ & $\mathrm{X}$ & \\
\hline Mikania sessilifolia DC. & $\mathrm{N} 1003$ & $\mathrm{X}$ & & & & \\
\hline Mikania smilacina DC. & N2619 & & & & & \\
\hline Mikania thapsoides DC. & R4873 & & & & & \\
\hline Mikania triphylla Spreng. ex Baker & N1320 & & & & & \\
\hline Mikania virgata B.L. Rob. & N485 & & & & & \\
\hline Mikania warmingii Sch. Bip. ex Baker & R2017 & & & & & \\
\hline Mikania sp. nov. * & N1741 & & & & & \\
\hline Praxelis grandiflora (DC.) Sch. Bip. & N296 & & & $\mathrm{X}$ & & \\
\hline Praxelis kleinioides (Kunth) Sch. Bip. & R3862 & & $\mathrm{X}$ & & & \\
\hline Pseudobrickellia brasiliensis (Spreng.) R.M. King \& H. Rob. & R2488 & $\mathrm{X}$ & $\mathrm{X}$ & & $\mathrm{X}$ & \\
\hline Pseudobrickellia sp. nov. & R1762 & & & & & \\
\hline Raulinoreitzia crenulata (Spreng.) R.M. King \& H. Rob. & R4148 & & & & & \\
\hline Stevia heptachaeta DC. & R922 & $\mathrm{X}$ & & $\mathrm{X}$ & & \\
\hline Stevia hilarii B.L. Rob. * & $\mathrm{R} 1025$ & & & & & \\
\hline Stevia myriadenia Sch. Bip. ex Baker & $\mathrm{R} 1948$ & $\mathrm{X}$ & & & & \\
\hline Stevia sp. nov. * & R1059 & & & & & \\
\hline Stomatanthes corumbensis (B.L. Rob.) R.M. King \& H. Rob. & R3963 & & & & & \\
\hline Stomatanthes dictyophyllus (DC.) R.M. King \& H. Rob. & R2833 & & & & & \\
\hline Stomatanthes pinnatipartitus (Sch. Bip. ex Baker) R.M. & & & & & & \\
\hline King \& H. Rob. & N1217 & & & & & \\
\hline Stomatanthes sp. nov. * & N513 & & & & & \\
\hline Symphyopappus compressus (Gardner) B.L. Rob. & N184 & $\mathrm{X}$ & $\mathrm{X}$ & & $\mathrm{X}$ & \\
\hline Trichogonia salviifolia Gardner & R604 & & $\mathrm{X}$ & $\mathrm{X}$ & & \\
\hline
\end{tabular}


Comparações com outros levantamentos recentes da família para uma determinada região ou localidade (figura 1) indicam que a Serra da Canastra possui o maior número de espécies e gêneros encontrados em uma única localidade (tabela 2). Entretanto, as diferenças nos números de gêneros e espécies levantados em cada localidade podem ser explicadas pelas diferenças no tamanho da área amostrada, bem como pela duração e peridiocidade das coletas, não revelando necessariamente uma maior riqueza florística.

A localidade do Pico das Almas na Bahia (Hind 1995) é potencialmente a mais rica em termos de

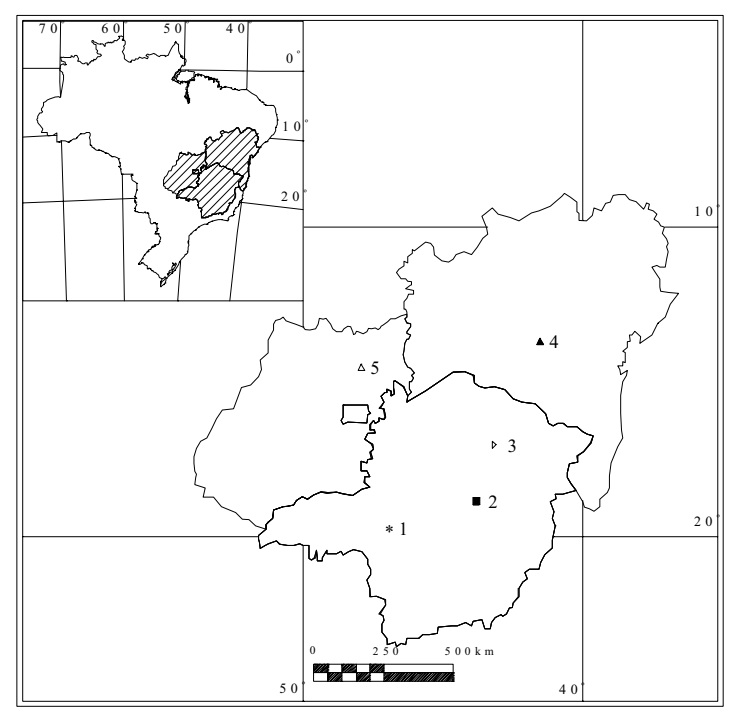

Figura 1. Localização geográfica aproximada do (1) Parque Nacional da Serra da Canastra, Minas Gerais; (2) Parque Nacional da Serra do Cipó, Minas Gerais; (3) Grão-Mogol, Minas Gerais; (4) Pico da Almas e Mucugê, Bahia; e (5) Parque Nacional da Chapada dos Veadeiros, Goiás. número de espécies por hectare, uma vez que possui apenas $24 \%$ da área do Parque Nacional da Serra da Canastra e conta com $60 \%$ do número total de espécies. Hind (1993) indica que o baixo número de espécies levantadas em Grão-Mogol, localizado na porção norte da Cadeia do Espinhaço provavelmente é em função do tamanho reduzido da área amostrada. Já o levantamento em Mucugê (Harley \& Simmons 1986) não deve refletir a sua verdadeira composição florística, devido principalmente as limitações impostas pela metodologia empregada, enquanto que os levantamentos florísticos da Serra do Cipó (Leitão Filho \& Semir 1987) e de Alto Paraíso (Munhoz \& Proença 1998), apesar de intensivos, provavelmente também não refletem o número total de espécies.

Apesar das limitações de realizar uma comparação florística adequada, a baixa porcentagem de espécies dessas regiões em comum com a Serra da Canastra (tabela 2) pode ser também devida a diferenças nos tipos de formações vegetacionais existentes em cada uma destas localidades (Giulietti \& Pirani 1988, Harley 1995, Munhoz \& Proença 1998), mas a comparação dos gêneros amostrados (tabela 2) mostra que a Serra da Canastra possui uma maior semelhança florística com a Chapada dos Veadeiros em Goiás, e com a Serra do Cipó e Grão-Mogol, ambas em Minas Gerais (mais de $70 \%$ dos gêneros são comuns a estas localidades), enquanto que com o Pico das Almas e com Mucugê a semelhança é menor (menos de 65\% dos gêneros em comum), indicando diferenças significativas na composição florística da Bahia (Harley 1995).

Estes levantamentos demonstram a grande importância relativa das Asteraceae na composição da vegetação de diferentes localidades, reforçando o fato de ser uma das mais ricas no Brasil (Barroso et al. 1991), e também uma das mais importantes no

Tabela 2. Principais levantamentos florísticos para a família Asteraceae realizados nos estados de Minas Gerais, Bahia e Goiás. Entre parênteses estão as porcentagens em relação ao total de cada localidade analisada.

\begin{tabular}{lccccc}
\hline Localidades & Gêneros $^{1}$ & Gêneros em comum & Espécies & Espécies em comum & Referência \\
\hline Serra da Canastra, MG & 66 & - & 215 & - & Nakajima 2000 \\
Serra do Cipó, MG & 48 & $37(77 \%)$ & 169 & $50(29 \%)$ & Leitão Filho \& Semir 1987 \\
Pico das Almas, BA & 56 & $36(64 \%)$ & 132 & $21(15 \%)$ & Hind 1995 \\
Alto Paraíso, GO & 47 & $39(82 \%)$ & 125 & $33(26 \%)$ & Munhoz \& Proença 1998 \\
Grão-Mogol, MG & 35 & $27(77 \%)$ & 70 & $19(27 \%)$ & Hind, não publicado \\
Mucugê, BA & 27 & $16(59 \%)$ & 56 & $4(7 \%)$ & Harley \& Simmons 1986
\end{tabular}

${ }^{1}$ Atualizado para os conceitos genéricos adotados no presente estudo, principalmente para Eupatorieae (King \& Robinson 1987) e Vernonieae (Robinson 1999). 
bioma dos cerrados, incluindo os campos rupestres (Mendonça et al. 1998). Além disso, o presente levantamento demonstra a grande importância da família Asteraceae na composição florística do Parque Nacional da Serra da Canastra, cuja flora é ainda pouco conhecida.

Agradecimentos - Os autores agradecem à Dra. Rosana Romero, coordenadora e executora do levantamento florístico; ao IBAMA - DF pela licença de pesquisa (processo 020015000086/93-37); ao Sr. Wagner Moreira de Lima e funcionários do IBAMA do PARNA Serra da Canastra pelo apoio logístico. O primeiro autor agradece à Diretoria de Pesquisa da Universidade Federal de Uberlândia e ao CNPq (processo 400491/96-6) pelo auxílio à pesquisa, aos assessores da revista pelas críticas e sugestões, e especialmente o Dr. Harold Robinson pela supervisão durante parte da pesquisa desenvolvida junto ao Smithsonian Institution, Washington DC, U.S.A.

\section{Referências bibliográficas}

BAKER, J.G. 1873-1884. Compositae. In Flora brasiliensis (C.F.P. Martius \& A.W. Eichler, eds.). F. Fleischer, Lipsiae. v.6, pars 2, p.1-374; pars 3, p.1-398.

BARROSO, G.M., PEIXOTO, A.L., ICHASO, C.L.F., COSTA, C.G., GUiMARÃES, E.F. \& LIMA, H.C. 1991. Sistemática de Angiospermas do Brasil. Editora Universitária UFV, Viçosa. v.3, p.237-258.

BREMER, K. 1994. Asteraceae. Cladistics and Classification. Timber Press, Portland.

BREMER, K. 1996. Major clades and grades of the Asteraceae. In Compositae: Systematics. Proceedings of the International Compositae Conference, Kew, 1994 (D.J.N. Hind \& H.J. Beentje, eds.). Royal Botanic Gardens, Kew, v.1, p.1-7.

DUBS, B. 1998. Prodromus Florae Matogrossensis. Part I. Checklist of Angiosperms. Part II. Types from Mato Grosso. The Botany of Mato Grosso. Series B, $n^{\circ} 3$. Betrona-Verlag, Küsnacht.

EITEN, G. 1983. Classificação da vegetação do Brasil. Conselho Nacional de Desenvolvimento Científico e Tecnológico, Brasília.

GOODLAND, R.J.A. 1979. Análise ecológica da vegetação do cerrado. In Ecologia do cerrado (R.J.A. Goodland \& M.G. Ferri, eds.). Livraria Itatiaia Editora Ltda. e EDUSP, Belo Horizonte e São Paulo, p.61-179.

GIUlietTI, A.M. \& PIRANI, J.R. 1988. Patterns of geographic distribution of some plant species from the Espinhaço Range, Minas Gerais and Bahia, Brazil. In Proceedings of a workshop on Neotropical Distribution Patterns (W.R.Heyer \& P.E.Vanzolini, eds.). Academia Brasileira de Ciências, Rio de Janeiro, p.39-69.

HARLEY, R.M. 1995. Introdução. In Flora of Pico das Almas - Chapada Diamantina, Bahia, Brazil. (B.L. Stannard, ed.). Royal Botanic Gardens, Kew, p.43-78.
HARLEY, R.M. \& SIMMONS, N.A. 1986. Florula of Mucugê, Chapada Diamantina, Bahia, Brazil. Royal Botanic Gardens, Kew.

HIND, D.J.N. 1993. New Compositae from the Serra do Grão-Mogol (Mun. Grão-Mogol, Minas Gerais, Brazil) and the surrounding area. Kew Bulletin 49:511-522.

HIND, D.J.N. 1995. Compositae. In Flora of Pico das Almas - Chapada Diamantina, Bahia, Brazil. (B.L. Stannard, ed.). Royal Botanic Gardens, Kew, p.175-278.

HIND, D.J.N. \& BEENTJE, H.J. 1996. Compositae: Systematics. Proceedings of the International Compositae Conference, Kew, 1994. Royal Botanic Gardens, Kew. v.1, p.621-626.

HOLMGREN, P.K, HOLMGREN, N.H. \& BARNETT, L.C. 1990. Index Herbariorum. Part I. The Herbaria of the World. 8 ed. The New York Botanical Garden, New York.

IBDF. 1981. Plano de manejo. Parque Nacional da Serra da Canastra. IBDF, Brasília.

KING, R.M. \& ROBINSON, H. 1987. The genera of the Eupatorieae (Asteraceae). Monographs in Systematic Botany. v.22. Missouri Botanical Garden, St. Louis.

LEITÃO FILHO, H.F. \& SEMIR, J. 1987. Compositae. In Flora da Serra do Cipó, Minas Gerais (A.M. Giulietti et al., coord.). Boletim de Botânica da Universidade São Paulo 9:29-41.

MENDONÇA, R.C., FELFILI, J.M., WALTER, B.M.T., SILVA, M.C., REZENDE, A.R., FILGUEIRAS, T.S. \& NOGUEIRA, P.E. 1998. Flora vascular do Cerrado. In Cerrado: ambiente e flora. (S.M. Sano \& S.P. Almeida, eds.). EMBRAPA - CPAC, Planaltina, DF, p.289-556.

MORAES, M.D. 1997. A família Asteraceae na planície litorânea de Picinguaba, município de Ubatuba, São Paulo. Dissertação de mestrado. Universidade Estadual Paulista, Rio Claro.

MUNHOZ, C.B.R. \& PROENÇA, C.E.B. 1998. Composição florística do município de Alto Paraíso de Goiás na Chapada dos Veadeiros. Boletim do Herbário Ezechias Paulo Heringer 3:102-150.

NAKAJIMA, J.N. 2000. A família Asteraceae no Parque Nacional da Serra da Canastra, MG. Tese de doutorado. Universidade Estadual de Campinas, Campinas.

RADFORD, A.E. 1986. Fudamentals of plant systematics. Harper \& Row, New York.

ROBINSON, H. 1999. Generic and subtribal classification of American Vernonieae. Smithsonian Contributions to Botany $n^{\circ} 89$, Washington, DC.

ROMERO, R. 2000. A família Melastomataceae no Parque Nacional da Serra da Canastra, MG. Tese de doutorado. Universidade Estadual de Campinas, Campinas.

ROMERO, R. \& NAKAJIMA, J.N. 1999. Espécies endêmicas do Parque Nacional da Serra da Canastra, MG. Revista Brasileira de Botânica 22:259-265.

ROQUE, N. \& PIRANI, J.R. 1997. Flora da Serra do Cipó, Minas Gerais: Compositae - Barnadesieae e Mutisieae. Boletim de Botânica da Universidade de São Paulo 16:151185.

SAINT HILAIRE, A.F.C.P. 1847. Voyage aux sources du Rio de S. Francisco. Arthus Bertrand, Paris, v.1. Viagens às nascentes do rio São Francisco. Tradução de Regina Regis Junqueira. 1975. Livraria Itatiaia Editora e EDUSP, Belo Horizonte e São Paulo. 